\title{
Invalidity of TCP.Theorem for Infinite-Component Fields
}

\author{
A. I. OKSAK and I. T. Todorov \\ Joint Institute for Nuclear Research, Moscow
}

Received June 27, 1968

\begin{abstract}
Necessary conditions are given for the TCP invariance of a local field with usual energy-momentum spectrum. Examples are constructed of both Fermi and Bose local free infinite-component fields which violate these conditions and consequently do not allow a TCP symmetry.
\end{abstract}

\section{Introduction}

It has been shown recently [1-4] that infinite-component local fields may violate the right connection between spin and statistics. The only example of TCP noninvariant infinite-component field known up to now, the Majorana field [5, 3, 4], is incompatible with usual spectrum conditions. Here we give examples of local infinite-component fields transforming under irreducible unitary representations of $S L(2, C)$ which satisfy usual spectrum conditions and violate TCP. These examples do not contradict Epstein's proof [6] of TCP in a theory of local observables, because his assumption of no mass degeneracy (with respect to spin) is not fulfilled in our case.

First of all, in Sect. 2, we derive some general properties of the twopoint function, valid also for tempered local infinite-component fields. The main tool in this investigation is the Bogolubov-Vladimirov theorem [7]. We give necessary conditions on the two-point function for the validity of TCP. We construct in Sect. 3 examples of free (FERMI and BOSE) fields of mass $m$ which violate these conditions. All our examples use the Majorana representations ${ }^{1}[0,1 / 2]$ and $[1 / 2,0]$ (but not Majorana equations).

\section{Two-Point Functions in Local and TCP-Invariant Theories}

We shall deal with the quantum field theory in which all Wightman axioms [9] except Lorentz invariance hold. We impose in particular (weak) locality and translation invariance including normal spectrum

1 For a detailed description of the Majorana representations the reader is referred to [4]. We are using here the notations $\left[l_{0}, l_{1}\right]$ of GEL'FAND and NATMARK [8] for the irreducible representations of $S L(2, C)$. 
condition. Locality implies

$$
\begin{aligned}
\langle 0|\varphi(x) \psi(y)-\varepsilon \psi(y) \varphi(x)| 0\rangle & =0 \quad \text { when } \quad(x-y)^{2}<0, \\
\varepsilon & = \pm 1 .
\end{aligned}
$$

We start with the formulation of Bogolubov-Vladimirov theorem [7] (we refer here to a part of the result of [7] only).

BV-Theorem. Let $F_{1}(x), F_{2}(x) \in S^{\prime}\left(R^{4}\right)$ be two tempered distributions satisfying the following conditions:

a) $\operatorname{supp} \widetilde{F}_{1}(p) \subset \bar{V}_{+}, \bar{V}_{+}$is the closed forward light cone,

$\operatorname{supp} \tilde{F}_{2}(p) \subset \bar{V}_{-}, \bar{V}_{-}=-\bar{V}_{+}$

b) $F_{1}(x)=F_{2}(x)$ for $x^{2}<0$

$$
\left(\tilde{F}_{1,2}(p)=\frac{1}{(2 \pi)^{2}} \int F_{1,2}(x) e^{-i p x} d^{4} x\right) .
$$

Then a function $F(z)$ exists holomorphic in the extended tube

$$
\mathscr{C}^{\prime}=\left\{z \in C^{4}: z^{2} \in \mathfrak{M}\right\}
$$

where $\mathfrak{M}$ is the complex plane with a cut along the real positive semiaxis, such that

Define

$$
\begin{aligned}
& F_{1}(x)=F(x+i 0)\left(\equiv \lim _{y \in V_{+}, y \rightarrow 0} F(x+i y)\right), \\
& F_{2}(x)=F(x-i 0) .
\end{aligned}
$$

and let

$$
\begin{aligned}
& W_{1}(x-y)=\left\langle 0\left|\varphi(x) \varphi^{*}(y)\right| 0\right\rangle, \\
& W_{\mathbf{2}}(x-y)=\left\langle 0\left|\varphi^{*}(x) \varphi(y)\right| 0\right\rangle
\end{aligned}
$$

$$
\varphi(x) \varphi^{*}(y)-\varepsilon \varphi^{*}(y) \varphi(x)=0 \quad \text { when } \quad(x-y)^{2}<0 .
$$

By the $\mathrm{BV}$-theorem we can deduce that there is a function $F(z)$ holomorphic in the extended tube $\mathscr{C}^{\prime}$ such that

$$
\begin{aligned}
& W_{1}(x)=F(x+i 0), \\
& W_{\mathbf{2}}(x)=\varepsilon F(-x-i 0) .
\end{aligned}
$$

The statistics associated with $\varphi(x)$ is connected with the question, whether $F(z)$ defined by (4), (2) is even or odd.

Proposition 1. If $F^{\prime}(z)$ defined by (4), (2) is even (resp. odd) in $\mathscr{C}^{\prime}$ :

$$
F(-z)= \pm F(z)
$$

then $\varepsilon$ in (3) is 1 (resp. - 1).

Proof. Suppose that $F(-z)=\lambda F(z), \lambda= \pm 1$. Due to (4) we have

$$
W_{2}(x)=\varepsilon F(-x-i 0)=\varepsilon \lambda F(x+i 0)=\varepsilon \lambda W_{1}(x),
$$

i.e. $W_{2}(x)-\varepsilon \lambda W_{1}(x)=0$. Now it is obvious that the equality $\varepsilon \lambda=-1$ is impossible since the sum of two positive-definite distributions $W_{2}(x)$ 
$+W_{1}(x)$ cannot be zero (otherwise $\| \varphi(f)|0\rangle\|=\| \varphi^{*}(f)|0\rangle \| \equiv 0$ ). This proves the assertion.

We give below the conversion of proposition 1 under the assumption of TCP-invariance (proposition 2). Let $\Theta$ be an anti-unitary operator such that

$$
\begin{aligned}
\Theta^{-1} \varphi(x) \Theta & =\eta(\varphi) \varphi^{*}(-x), \quad|\eta(\varphi)|=1, \\
\Theta^{-1} \varphi^{*}(x) \Theta & =\eta\left(\varphi^{*}\right) \varphi(-x) ;
\end{aligned}
$$

taking hermitian conjugation of (5) one obtains

$$
\eta\left(\varphi^{*}\right)=\overline{\eta(\varphi)} \text {. }
$$

It is easily verified that TCP-invariance imposes the following condition on the two-point functions

$$
W_{\varphi, \psi}(x) \equiv\left\langle 0\left|\varphi\left(\frac{x}{2}\right) \psi\left(-\frac{x}{2}\right)\right| 0\right\rangle=\overline{\eta(\varphi)} \overline{\eta(\psi)} W_{\psi, \varphi}(x) .
$$

Let us mention that if $W_{\varphi, \varphi} \neq 0$ (resp. $W_{\varphi, \psi} \neq 0$ ) then (6) implies $\eta^{2}(\varphi)=1$ (resp. $\eta(\varphi) \eta(\psi)= \pm 1$ ). Let $G(z)$ be the analytic function in $\mathscr{C}^{\prime}$ defined by

$$
\begin{aligned}
G(x+i 0) & =W_{\varphi, \psi}(x), \\
G(-x-i 0) & =\varepsilon W_{\psi, \varphi}(x)
\end{aligned}
$$

according to the $\mathrm{BV}$-theorem. TCP invariance leads to the identity

$$
G(-z)=\varepsilon \eta(\varphi) \eta(\psi) G(z) .
$$

Indeed, the functions

$$
G_{1}(z)=G(-z), \quad G_{2}(z)=\varepsilon \eta(\varphi) \eta(\psi) G(z)
$$

are analytic in $\mathscr{C}^{\prime}$ and due to (7), (6) their boundary values from the forward tube coincide:

$$
G_{1}(x+i 0)=G_{2}(x+i 0) .
$$

In particular taking $\psi=\varphi^{*}$ in (8) we obtain

\section{Proposition 2. If}

a) $\left\langle 0\left|\varphi(x) \varphi^{*}(y)-\varepsilon \varphi^{*}(y) \varphi(x)\right| 0\right\rangle=0$ when $(x-y)^{2}<0$,

b) there exists TCP symmetry

then $F(z)$ defined by (4), (2) satisfies

$$
F(-z)=\varepsilon F(z)
$$

\section{Examples of Infinite-Component Fields Violating TCP-Theorem}

Let us consider one of the Majorana representations $[0,1 / 2]$ or $[1 / 2,0]$ acting in the Hilbert space $X$. These are the only irreducible representations of $S L(2, C)$ for which a 4 -vector of (unbounded) hermitian operators 
$L_{\mu}$ can be defined such that

$$
V(A) L_{\mu} V\left(A^{-1}\right)=L_{\nu} \Lambda(A)_{\mu}^{\nu} .
$$

The operator $L_{0}$ is positive definite (its eigenvalues being $s+1 / 2$, $s=l_{0}, l_{0}+1, l_{0}+2, \ldots ; l_{0}$ is 0 or $1 / 2$ ). We define the free field $\psi$ as a linear operator valued functional of the vector $f \in X$ satisfying the anticommutation relations:

where

$$
\begin{aligned}
& {[\psi(x, f), \psi(y, g)]_{+}=\left[\psi(x, f)^{*}, \psi(y, g)^{*}\right]_{+}=0,} \\
& {\left[\psi(x, f)^{*}, \psi(y, g)\right]_{+}=\left(f\left|\frac{1}{m} L_{\mu} \frac{\partial}{\partial x_{\mu}}+\frac{1}{i} c\right| g\right) \cdot D_{m}(x-y)}
\end{aligned}
$$

$$
0<c<1 / 2 \text {. }
$$

The field $\psi$ is characterized completely by the two-point functions

$$
\begin{aligned}
& \left\langle 0\left|\psi(x, f)^{*} \psi(y, g)\right| 0\right\rangle=\left(f\left|\frac{1}{m} L_{\mu} \frac{\partial}{\partial x_{\mu}}+\frac{1}{i} c\right| g\right) \cdot D_{m}^{-}(x-y), \\
& \left\langle 0\left|\psi(x, g) \psi(y, f)^{*}\right| 0\right\rangle=\left(f\left|\frac{1}{m} L_{\mu} \frac{\partial}{\partial x_{\mu}}-\frac{1}{i} c\right| g\right) \cdot D_{m}^{-}(x-y) .
\end{aligned}
$$

All other two-point functions as well as the higher order truncated vacuum expectation values are zero. It can be checked in a straightforward way that the theory so defined satisfies all Wightman axioms (except finite componentness of the field). The positive definiteness of the metric in the Hilbert space of states follows from the positive definiteness of $L_{0}$ which implies

$$
L_{\mu} p^{\mu} \geqq m\left(l_{0}+1 / 2\right) \quad \text { for } \quad p^{0}=\sqrt{m^{2}+\boldsymbol{p}^{2}} \quad\left(l_{0}=0,1 / 2\right) .
$$

We can give explicitely the action of the field $\psi$ in Fock space by decomposing it in terms of creation and annihilation operators of particles with fixed momentum $\boldsymbol{p}$ and spin $s$ :

$$
\begin{aligned}
& \psi(x, f)=\frac{1}{\sqrt{2} \cdot(2 \pi)^{3 / 2}} \sum_{s=l_{0}}^{\infty} \int_{p_{0}=\omega} \sum_{\zeta=-s}^{s}\left\{\left(s+\frac{1}{2}-c\right)^{1 / 2} \cdot a_{s \zeta}(\boldsymbol{p})\right. \\
& \left.\cdot e^{-i p x}+\left(s+\frac{1}{2}+c\right)^{1 / 2} \cdot b_{s,-\zeta}^{*}(\boldsymbol{p}) e^{i p x}\right\} \cdot\left(u_{s \zeta}(\boldsymbol{p}), f\right) \frac{d^{3} \boldsymbol{p}}{p^{0}}
\end{aligned}
$$

where $\omega=\omega(\boldsymbol{p})=\left(m^{2}+\boldsymbol{p}^{2}\right)^{1 / 2}$ and $u_{s \zeta}(\boldsymbol{p})$ is normalized eigenvector of the total spin and its third projection (with eigenvalues $s$ and $\zeta$ ) which can be written as

$$
\left.u_{s \zeta}(\boldsymbol{p})=V\left(B_{p}\right) \mid s, \zeta\right) .
$$

Here $V\left(B_{p}\right)$ is the representation of the "boost" transformation

$$
B_{p}=\left(\omega+m+\sigma_{j} p^{j}\right) \cdot[2 m(\omega+m)]^{-1 / 2} .
$$


Due to (10) and (11) $a_{s \zeta}^{(*)}$ and $b_{s \zeta}^{(*)}$ have to satisfy the canonical anticommutation relations

$\left[a_{s \zeta}(\boldsymbol{p}), \quad a_{s^{\prime} \zeta^{\prime}}^{*}(\boldsymbol{p})\right]_{+}=\left[b_{s \zeta}(\boldsymbol{p}), \quad b_{s^{\prime} \zeta^{\prime}}^{*}(\boldsymbol{q})\right]_{+}=\delta_{s s^{\prime}} \delta_{\zeta \zeta^{\prime}} \omega(\boldsymbol{p}) \delta(\boldsymbol{p}-\boldsymbol{q})$

(all other anticommutators vanishing identically). We assume here the standard Fock representation of the canonical anticommutation relation in a Hilbert space $\mathscr{H}$ with an unique vacuum state $|0\rangle$ such that

$$
a_{s \zeta}(\boldsymbol{p})|0\rangle=b_{s \zeta}(\boldsymbol{p})|0\rangle=0 .
$$

Eqs. (10) and (11) are a consequence of (12)-(16) because of the identities

$$
\begin{aligned}
\sum_{s \zeta}\left(s+\frac{1}{2}\right)\left(f\left|V\left(B_{p}\right)\right| s \zeta\right)\left(s \zeta\left|V\left(B_{p}\right)^{*}\right| g\right) \\
=\left(f\left|V\left(B_{p}\right) L_{0} V\left(B_{p}\right)^{*}\right| g\right)=\frac{1}{m}\left(f\left|L_{\mu} p^{\mu}\right| g\right) .
\end{aligned}
$$

On the other hand the field $\psi$ with vacuum expectation values (10), (11) clearly does not allow a TCP symmetry operation of the type (5) because the necessary condition for TCP-invariance (6) is violated. The function $F(z)$ defined by $\mathrm{BV}$-theorem in this case has the form

$$
F(z)=(f \mid g) F_{0}\left(z^{2}\right)+z^{\mu}\left(f\left|L_{\mu}\right| g\right) F_{1}\left(z^{2}\right)
$$

and hence is neither odd nor even in contradiction with (9). (Obviously for $c=0$ the even term $F_{0}$ vanishes and the field becomes TCP invariant.) We observe that though the field (12) is not TCP invariant it describes particles and antiparticles with equal masses.

We mention that each term in the sum (12) with fixed $s$ represents a non-local field. For the compensation of the non-local terms in the total anticommutator the mass degenerary seems to be essential. As suggested in [2] it is interesting to note that a theory with an infinitely degenerate mass level (with respect to spin) violates the compactness condition proposed by HaAG and SwIECA [10].

We see from the above example that TCP violation is not necessarily related to the wrong connection between spin and statistics: $\psi$ is a local Fermi, field describing half-integer spin particles for the representation $[1 / 2,0]$ and integer spin particles for the representation $[0,1 / 2]$. We can construct just as well an example of a free Bose TCP non-invariant field $\varphi$. For this aim we define the commutator of $\varphi$ and $\varphi^{*}$ by $^{2}$

$$
\begin{aligned}
{\left[\varphi(x, f)^{*}, \varphi(y, g)\right] } & =\left(f\left|i\left(\frac{1}{m} L^{\mu} \partial_{\mu}\right)^{2}-\frac{c}{m} L^{\mu} \partial_{\mu}\right| g\right) \cdot D_{m}(x-y), \\
& 0<c<1 / 2
\end{aligned}
$$

2 This example was constructed in a discussion of the second named author with Prof. I. Bialynicki-Birula. 
(the commutator between two $\varphi$ vanishing identically). It is easy to check that the free field $\varphi$ so defined satisfies all requirements of local quantum field theory (except finite componentness) and violates TCP.

On the other hand, it is not difficult to construct examples of Poincaré and TCP invariant infinite-component fields (satisfying the spectrum conditions) which are not weakly local (see e.g. [11]).

It is a pleasure to thank Prof. I. BIALynickr-Binula for an useful discussion.

\section{References}

1. Feldman, G., and R. T. Matthews: Ann. Phys. (N. Y.) 40, 19 (1966). ; Phys. Rev. 151, 1176 (1966); 154, 1241 (1967).

2. Streater, R. F.: Commun. Math. Phys. 5, 88 (1967).

3. Abers, E., I. T. Grodsky, and R. E. Norton: Phys. Rev. 159, 1222 (1967).

4. Stoyanov, D. T., and I. T. Todorov: Self-coupled representations of the Lorentz-group and infinite component Fermi fields. ICTP, Trieste, preprint IC/67/58 (1967).

5. Majorana, E.: Nuovo Cimento 9, 335 (1932).

6. Erstein, H.: J. Math. Phys. 8, 750 (1967).

7. Bogolubov, N. N., and V. S. Vladimirov: Nauchnye Dokl. Vysshei Shkoly N. 3, 26 (1958) and N. 2, 179 (1959).

8. Nammark, M. A.: Linear representations of the Lorentz group, London: Pergamon Press 1964.

Gel'fand, I. M., R. A. Minlos, and Z. Ya. Shapiro: Representations of the rotation and Lorentz groups and their applications. London: Pergamon Press 1963.

9. Streater, R. F., and A. S. Wightman: PCT, spin and statistics and all that. New York-Amsterdam: W. A. Benjamin, Inc. 1964.

Jost, R.: The general theory of quantized fields. Providence, Rhode Island: Amer. Math. Soc. 1965.

10. HaAg, R., and J. Swieca: Commun. Math. Phys. 1, 308 (1965).

11. Todonov, I. T., and R. P. ZAIKov: Spectral representation of the covariant two-point function and infinite-component fields with arbitrary mass spectrum. ICTP, Trieste Internal Report IC/68/50 (1968).

\section{A. I. OKSAK}

Joint Institute for Nuclear Research

Head Post Office Box 79

Moscow, UdSSR

Present address :

Institute for High Energy Physics

Serpukhov, UdSSR

\section{T. ToDorov}

Joint Institute for Nuclear Research Head Post Office

P.O. Box 79

Moscow, UdSSR

After September 6, 1968:

Institute for Advanced Study

Princeton, New Jersey 08540, USA 